\title{
Study of Sharing Patient Information by Nurses Between Inpatient and Outpatient Wards in Japan
}

\author{
Natsuko NISHIDA ${ }^{\mathrm{a}, 1}$, Tomoko HIKITA ${ }^{\mathrm{a}}$, Megumi Iida ${ }^{\mathrm{a}}$, \\ Goshiro Yamamoto ${ }^{\mathrm{a}}$ and Tomohiro Kuroda ${ }^{\mathrm{a}}$ \\ ${ }^{\text {a Kyoto University }}$
}

\begin{abstract}
Shortening hospital stays increases communication needs between nurses in inpatient and outpatient wards. Smooth information sharing is required to reduce the workload of nurses and improve the quality of patient care. However, electronic medical records (EMR) system does not have sufficient functions to support information sharing between wards, because EMR has been developed mainly for recording. This study led to three improvements; unified communication tool, common patient list linked to EMR, and outpatient nursing diagnosis.
\end{abstract}

Keywords. Collaboration support, electronic medical record, patient information, information sharing

\section{Introduction}

In Japan, many activities $[1,2]$ are conducted to shorten the hospital stay, which was, as widely known, longer than other countries [3]. As a result, some of the care and guidance that was provided in inpatient wards during hospitalization is now provided in outpatient wards. For example, the instructions on how to lead daily lives and how to use medical equipment are given before discharge, and the evaluations on their management are performed in the outpatient ward. Therefore, the importance of information sharing between inpatient and outpatient wards increases for better clinical outcome due to the shortening the hospital stay. The nurses at least need to know when a particular patient is admitted to discharged, and how the patient is instructed.

Since the main functions of electronic medical records (EMR) system is to accumulate information on patients' conditions over time, they do not have enough functions to support information sharing between nurses. Therefore, nurses develop their own ways to share the information between inpatient and outpatient wards.

In this study, we aim to clarify the current information sharing methods by conducted a survey with nurses. Based on the results of the survey, we discussed what kind of functions would enable efficient information sharing between inpatient and outpatient wards over EMR.

\footnotetext{
${ }^{1}$ Corresponding Author, Natsuko Nishida, Kyoto University.
} 


\section{Methods}

From September to December 2019, we conducted an interview survey on the means and types of information used to share patient information between nurses in different wards, as well as problems faced in information sharing. These two departments have a long course of chronic diseases, and many patients are repeatedly admitted and discharged. Therefore, in this survey, we focus on the two departments, I and II, then our targets were two nursing sections, $\mathrm{A}$ and $\mathrm{B}$, related to the inpatient wards of a hospital and their outpatient ward as section C. The participants were one head nurse and one vice head nurse from each section, additionally plus two nurses from section $\mathrm{C}$. The basic information of each section is following:

- Section A (inpatient wards, department I): 59 beds, 45 nurses

- Section B (inpatient wards, department II): 53 beds, 35 nurses

- Section C (outpatient ward): 1336 patients per day (department I: 174 patients, II: 129 patients) average, Sep. 2019), two departments (corresponding to I and II)

In addition, this study was conducted with the approval of the ethics committee of Nursing Department of Kyoto University Hospital.

\section{Results and Discussions}

The responses from each department are as follows. Means for information sharing: Some departments in the hospital as a whole share information through the "shared folder" function, which can be accessed only by authorized personnel of paper or electronic medical records. However, in these two departments, information about patients between the wards and outpatients is shared between vice head nurses of each department by phone or messaging service of EMR system.

The information includes next hospital visits or date of admission, the care that needs to be continued, and the status of the patient and his/her family. These are recorded in the EMR, but the main points are summarized and communicated verbally.

Problem (outpatient ward): To find particular patient who is handed over by inpatient wards from patient list is time consuming. As the way of information sharing varies among wards, to summarize and understand the patient is also time consuming. For example, some wards creating a file outside of EMR to record statuses of patients who need care. Moreover, our nursing record in outpatient ward is forced to record in common progress note. Therefore, we cannot use search function implemented for the independent nursing record which is used in inpatient ward, it is difficult for ourselves to collect nursing problems.

Problems (inpatient wards): Nursing records in the outpatient ward are written in the common progress report page. Therefore, only the way to collect nursing problems is to read the progress report manually. As may patients have long medical histories, it is quite time consuming.

In the inpatient wards, there are multiple places to record information, such as the nursing database (basic patient information) for organizing patient information and nursing diagnosis plan, and the progress record or SOAP for evaluating assessment and planning. On the other hand, since only the SOAP screen is used in the outpatient ward, 
nurses in the outpatient ward and the inpatient ward find it difficult to collect information because the screens they mainly use are different.

Through above discussion, we concluded that the following functions are indispensable for smooth information sharing between inpatient and outpatient wards.

1. To unify a tool for the collection of information. The tool should be linked to the nursing record and have the ability to be referred by all nurses in the inpatient and outpatient wards.

2. To have a common patient list linked to EMR. The list should have the ability to register the date and time of care, the status (new, ongoing, done, etc.), and to link to the SOAP describing the care. It should be possible to refer to the summarised medical history, the date of the next visit or admission, the care that needs to be continued, and the status of the patient and his/her family.

3. To use nursing diagnosis function in outpatient wards. The function should be able to share nursing problems, planning dates, specific interventions, and patient achievement with the inpatient wards.

\section{Conclusions}

Conventionally, functions of EMR system have been developed individually for inpatient and outpatient wards. However, in order to shorten the length of hospital stay and to improve patient care, there is a need for a function that allows both inpatient and outpatient wards to cooperate in providing care to patients. With the help of some nurses in each ward, we investigated current information sharing methods and their problems, then found three improvement points; adding functions such as a unified communication tool, a common patient list linked to medical record information, and nursing diagnosis in outpatient settings. We have not yet investigated the interference of the additional functions in this work, so that further investigation of the problems through the design and implementation of the specific functions will be future work.

\section{Acknowledgement}

We would like to thank the nurses in the two inpatient wards and the outpatient ward who cooperated in our survey.

\section{References}

[1] Seido M, Shinohara A. Reducing length of stay in rehabilitation hospital after stroke by refining the rehabilitation program. Rehabilitation Science. Vol. 3, No. 4, pp. 54-58, 2018.

[2] Akihiro S et al. Clinical approach to shortening length of hospital stay in elderly patients with acute heart failure requiring intensive care. Circulation Reports, CR-19-0132, Released January 29, 2020.

[3] OECD data. Length of hospital stay. https://data.oecd.org/healthcare/length-of-hospital-stay.htm

[4] Rouleau $\mathrm{G}$ et al. Impact of information and communication technologies on nursing care: Results of an overview of systematic reviews. JMIR, Vol. 19, Issue 4, pp. 1-21, 2017. 